\title{
A new and fast method for the creation of 3D micro and nano structures
}

\author{
J.J.L. Mulders*, J.S. Faber* \\ *FEI Electron Optics, Achtseweg Noord 5, 5600 KA Eindhoven, The Netherlands
}

For the creation of nano structures several techniques, based on charged particle irradiation are available. Examples are Electron Beam Lithography, FIB milling and deposition and Electron Beam Induced Deposition (EBID. The scientific developments of EBID include a search for better purity of the deposited material including some post processing capabilities. Only recently it has been shown that directly deposited (Co) material can have a good purity and as a consequence a desired, well defined property at the nano scale [1]. The EBID technique has a good potential for small structures down to sub $10 \mathrm{~nm}$, but the application to larger areas is not very practical due to the excessively long exposure times. FIB milling and deposition are faster, but require the elimination of the influence of the $\mathrm{Ga}^{+}$implanted particles. Although FIB milling can easily be applied for $3 \mathrm{D}$ structuring some issues such as local charging, local channeling and re-deposition can be a real roadblock for the creation of nano and micro scale structures.

A novel in-situ method has been developed for the direct creation of micro and nano structures within an electron microscope / DualBeam. The method mainly uses standard components, but they are driven in a new parameter space. The main characteristics are a very high speed (compared to standard techniques such as IBID and EBID) and a simplified, true 3 dimensional capability including mushroom shapes, overhang, free floating structures and closed structures. Including a direct SW control for the complete creation process, this new method has the potential of translating 3D CAD design into a CAM based process with a mix of both large and small structures as well as a variety of materials. The new method is most efficient in the micron and sub micron range, but can easily combined with standard EBID technology, covering the total range from sub $10 \mathrm{~nm}$ up to over 100 um within a practical time frame for creation and all in one tool.

[1] A Fernandez-Pacheco, J M De Teresa, R Cordoba, M R Ibarra1, J. Phys. D: Appl. Phys. 42 (2009) 055005 